\title{
Controlled challenge experiment demonstrates substantial additive genetic variation in resistance of Nile tilapia (Oreochromis niloticus) to Streptococcus iniae
}

Benjamin R. LaFrentz ${ }^{a, *}$, Carlos A. Lozanob, Craig A. Shoemaker ${ }^{a}$, Julio C. García ${ }^{a}$, DeHai Xu' ${ }^{\mathrm{a}}$, Marie Løvoll ${ }^{\mathrm{c}}$, Morten Rye

${ }^{a}$ United States Department of Agriculture-Agricultural Research Service (USDA-ARS), Aquatic Animal Health Research Unit, 990 Wire Road, Auburn, AL, 36832-4352, USA

${ }^{b}$ Akvaforsk Genetics Center AS, Sjфlsengvegen 22, N-6600 Sunndalsøra, Norway

${ }^{c}$ VESO Vikan, Beisvågveien 107, Vikan, N-7810 Namsos, Norway.

* Corresponding author: Tel.: +1 334887 3741; fax: +1 334887 2983. Email address:

benjamin.lafrentz@ars.usda.gov (B. LaFrentz) 


\section{ABSTRACT}

Streptococcus iniae is an etiologic agent of streptococcal disease in tilapia and is one of several Streptococcus spp. that negatively impact worldwide tilapia production. Methods for the prevention and control of $S$. iniae include vaccines, management strategies, and antibiotics. A complimentary preventative approach may include selective breeding for disease resistance, but the potential for this is unknown in tilapia. This study was initiated to challenge Nile tilapia (Oreochromis niloticus) families for estimation of genetic parameters for resistance to S. iniae. A total of 143 full- and paternal half-sib families (avg. $176 \mathrm{~g}, \mathrm{sd}=50 \mathrm{~g}$ ) were divided into two groups with each containing on average 9 fish per family. The challenge was designed with the intent to utilize fish injected with $S$. iniae (Group 1) as shedder fish to transfer the bacterium to cohabitated fish (Group 2). Tilapia from Group 1 were challenged by intraperitoneal injection with a volume containing $1.15 \times 10^{8}$ colony-forming units $S$. iniae per fish, and then cohabitated with tilapia from Group 2 in a single tank. Accumulated mortality at the end of the experiment was $60 \%$ for the fish challenged by injection and $6.4 \%$ for fish challenged by cohabitation. The results revealed high variation for mean survival of the families injected with S. iniae (range from $0 \%$ to $100 \%, \mathrm{CV} 69 \%$ ). The estimated heritability of post-challenge survival in Group 1 was $0.42 \pm 0.07$ on the observed binary scale and $0.58 \pm 0.09$ on the underlying liability scale, derived from fitting a linear animal model and a sire-dam threshold model, respectively. In summary, substantial additive genetic variation in resistance to $S$. iniae was observed when fish were challenged by injection, and this suggests promise for genetic improvement of tilapia for resistance to $S$. iniae through selective breeding.

Keywords: Streptococcus iniae; Nile tilapia; Oreochromis niloticus; selective breeding; disease resistance; heritability 


\section{Introduction}

Tilapia (Oreochromis spp.) production from the aquaculture sector was reported to be 3,197,330 $\mathrm{t}$ with a value of $\$ 5.2$ billion in 2012 (FAO, 2014) and is expanding (Fitzsimmons et al., 2011). Intensification of tilapia production has resulted in disease outbreaks that negatively affect production (Shoemaker et al., 2006). Streptococcus iniae is a non-groupable Streptococcus sp. (Pier and Madin, 1976) and the economic impact of this pathogen on tilapia aquaculture has been realized for more than a decade ( Shoemaker et al., 2001; Agnew and Barnes, 2007). Methods of prevention and control for S. iniae include vaccination (Eldar et al., 1997; Locke et al., 2010; Shoemaker et al., 2010; LaFrentz et al., 2011), management strategies such as a reduction of fish density (Shoemaker et al., 2000), and use of antibiotics (Gaunt et al., 2010). Effective control and prevention of streptococcosis in the future will likely require an integrated fish-health management approach consisting of all these methods.

Selective breeding for resistance to disease is a complimentary strategy to improve health and performance in tilapia. Research has demonstrated that resistance to infectious disease can be moderately heritable depending on the host and pathogen (Silverstein et al., 2009; Ødegård et al., 2011; Evenhuis et al., 2015). Therefore, there is potential for genetic improvement and research has illustrated this concept (Ødegård et al., 2011; Wiens et al., 2013). The potential for selectively breeding tilapia for resistance to $S$. iniae is unknown; therefore, this study was initiated to challenge Nile tilapia (O. niloticus) families for estimation of genetic parameters for resistance to $S$. iniae.

\section{Materials and methods}

\subsection{Fish and rearing conditions}

Fish used for this study were from the third generation of the Spring Genetics Nile tilapia 
breeding program with nucleus operations in Homestead, Florida, US. Genetic material used to produce the base population of Spring Genetics had undergone 12 generations of selection and was introduced to Spring Genetics in 2010. The base population at Spring Genetics was derived from a selective breeding program in Nicaragua, with three generations of selection primarily focused on growth rate and fillet yield (Gjerde et al., 2012). The program conducted in Nicaragua was designed and supervised by Akvaforsk Genetics Center, Norway. The material originated from a Vietnamese family-based breeding program started in 1999 that had been genetically improved for growth in freshwater earthen ponds for four cycles of selection. This Vietnamese material originated from the fifth generation of the Genetically Improved Farmed Tilapia (GIFT) breeding project conducted in the Philippines (Bentsen et al., 2012) and local Vietnamese strains. The collaborative GIFT research project was started in the Philippines in 1989 as a complete diallel cross experiment carried out with eight strains of Nile tilapia from Africa and Asia (Bentsen et al., 1998).

A total of 162 full- and paternal half-sib families were produced in 2014 using 93 sires and 162 dams selected for increased harvest weight for two generations in the US. Families were produced during a period of $16 \mathrm{~d}$ by natural mating in single pair breeding units. A nested design was used where each male was mated with two different females. Fry or fertilized eggs from each female were collected, placed in individual incubators and after five days, an average of 215 fry from each family were randomly selected and transferred to individual nursery tanks where they were kept for approximately nine days. Then fish from each family were stocked in separate rearing units (144 tanks and 18 hapas) until they reached a size allowing for physical tagging with passive integrated transponder (PIT) tags (Biomark GPT12).

At a mean age of $84 \mathrm{~d}$ after egg collection, fish from all families were PIT tagged, 
individually weighed (avg. $62 \mathrm{~g}, \mathrm{sd}=22 \mathrm{~g}$ ) and representatives of all families were stocked in separate test units for local performance testing, or holding tanks destined for challenge testing. Fish from one of the holding tanks, originating from 143 families, were individually weighed (avg. $176 \mathrm{~g}, \mathrm{sd}=50 \mathrm{~g}$ ) and randomly separated into two groups (Group 1 and Group 2) with equal number of fish per group (on average nine fish per family per group). These two groups were transported to the Aquatic Animal Health Research Unit (AAHRU) by truck following protocols previously developed.

\subsection{Fish rearing conditions at $A A H R U$}

Upon arrival at the AAHRU, the Group 2 fish were stocked into a single 5,550 L tank, while Group 1 fish were distributed into seven $350 \mathrm{~L}$ troughs. The large tank and troughs were supplied with $\sim 26{ }^{\circ} \mathrm{C}$ dechlorinated municipal water at approximate flow rates of 13 and $2 \mathrm{~L}$ $\min ^{-1}$, respectively. Fish were acclimated for $12 \mathrm{~d}$ prior to bacterial challenge and fed daily (23\% body weight) with Aquamax Grower feed (PMI Nutrition International, Inc., Brentwood, MO, USA). The PIT tag number and date of death was recorded using an ISO handheld reader (ARE H5; AEG ID, Ulm, Germany) for mortalities that occurred during the acclimation period. The tilapia family information and PIT tag numbers were not provided to AAHRU in order for the experiment to be blinded. All procedures utilizing fish were approved by the USDA-ARS, AAHRU Institutional Animal Care and Use Committee.

\subsection{Streptococcus iniae growth conditions and passage through fish}

A virulent strain of $S$. iniae, ARS-98-60, was used for the bacterial challenge. This isolate was recovered from hybrid striped bass (Morone saxatilis $\times$ M. chrysops) with natural 
streptococcal disease (Shoemaker et al., 2010) and identified by species specific PCR (Mata et al., 2004). The $S$. iniae isolate was resuscitated from a $-80{ }^{\circ} \mathrm{C}$ glycerol stock and grown for $24 \mathrm{~h}$ at $28^{\circ} \mathrm{C}$ with shaking at $140 \mathrm{rpm}$ in tryptic soy broth (TSB; BD, Sparks, MD, USA) (Shoemaker et al. 2006). This culture was used to pass the isolate through fish. Briefly, $100 \mu \mathrm{L}$ of the $24 \mathrm{~h}$ culture was injected into the peritoneal cavity (i.p. injection) of five tilapia (mean weight, $54 \mathrm{~g}$ ). At $24 \mathrm{~h}$ post-injection, brain tissues from moribund fish were aseptically plated onto sheep blood agar (SBA; Remel, Lenexa, KS, USA) and incubated at $28{ }^{\circ} \mathrm{C}$ for 48 h. A colony of $S$. iniae recovered from fish was passed from the SBA plate into TSB, incubated, and used to inject another group of tilapia as described above. This process was repeated one additional time for a total of three passages through tilapia. After the final passage, a colony was passed into TSB, grown, and then $20 \%$ glycerol stocks were prepared and frozen at $-80^{\circ} \mathrm{C}$. The isolate was confirmed as $S$. iniae by PCR.

The frozen stock of $S$. iniae generated following passage through tilapia was used for the challenge. Briefly, $100 \mu \mathrm{L}$ of thawed stock was inoculated into each of four flasks containing 50 $\mathrm{ml}$ TSB and then incubated at $28{ }^{\circ} \mathrm{C}$ with shaking at $140 \mathrm{rpm}$. After $24 \mathrm{~h}$ of growth, each of the four cultures were aseptically combined into a single flask, the optical density (OD) measured at $540 \mathrm{~nm}$ was determined to be 1.35 , and this culture was used for the bacterial challenge. The number of viable colony-forming units $(\mathrm{cfu}) \mathrm{mL}^{-1}$ was determined by spread plating $100 \mu \mathrm{L}$ volumes of 10-fold serial dilutions (in duplicate) onto SBA. Plates were incubated for $48 \mathrm{~h}$ at $28^{\circ} \mathrm{C}$, and colonies were counted and averaged to enumerate the cfu $\mathrm{mL}^{-1}$ using standard procedures.

\subsection{Streptococcus iniae challenge}


The average weight of fish was approximately $176 \mathrm{~g}(\mathrm{sd}=50 \mathrm{~g})$ and this was determined $12 \mathrm{~d}$ prior to bacterial challenge (see section 2.1.). The challenge was designed with the intent to utilize fish injected with $S$. iniae (Group 1) as shedder fish to transfer the bacterium to cohabitated fish (Group 2) and determine the mortality of each family by both injection and cohabitation. To accomplish this, tilapia from Group 1 were injection challenged with S. iniae, and then cohabitated with tilapia from Group 2. Groups of 20-40 tilapia from Group 1 were anesthetized with 90 ppm tricaine methane sulfonate (MS-222; Argent Chemicals, Redmond, WA, USA) and injected ip with $100 \mu \mathrm{L}$ of the $S$. iniae culture using a 25 gauge $5 / 8$ inch needle. Each fish received $1.15 \times 10^{8} \mathrm{cfu}$, a dose that resulted in $\sim 60-70 \%$ mortality in preliminary experiments (data not shown). Prior to injection, the PIT tag number of each fish was recorded. Following injection, fish were placed into the 5,550 L tank containing fish from Group 2 and this process was repeated for $3 \mathrm{~h}$ until all fish from Group 1 were challenged and stocked into the tank. Following challenge, dead fish were removed twice daily for $45 \mathrm{~d}$ and the PIT tag numbers and date of death were recorded. Re-isolation of S. iniae was attempted daily from a minimum of $20 \%$ of the dead fish by inoculating brain tissue onto SBA. Plates were incubated at $28^{\circ} \mathrm{C}$ for 48 $\mathrm{h}$ and examined for the presence of beta-hemolytic colonies indicative of $S$. iniae. Upon termination of the challenge, all surviving fish were euthanized, examined for clinical signs of disease, and PIT tag numbers were recorded. Water quality parameters were determined over the course of the $45 \mathrm{~d}$ challenge. Dissolved oxygen (DO) and temperature were measured with a ProODO meter (Yellow Springs Instruments, Yellow Springs, OH, USA). Nitrate, ammonia, hardness, and alkalinity were determined using a Hach CEL/890 Advanced Portable Laboratory (Loveland, CO, USA) according to manufacturer's directions. 


\subsection{Data Analysis}

Survival at the end of the challenge (scored as 1 for alive fish and 0 for dead fish) was analyzed for both Group 1 and Group 2. Since some mortality occurred during acclimation, families with less than five fish at the onset of the study were not included in the analysis (10 families excluded from Group 1 and six families excluded from Group 2). A total of 1,116 records were used for Group 1 (from 133 families) and 1,226 for Group 2 (from 137 families).

Univariate animal linear (1) and sire-dam threshold (2) models were used to obtain estimates of variance components of random effects for survival by Restricted Maximum Likelihood (REML). Of the two, the threshold models offer the most accurate estimations as they treat binary survival scores as normally distributed observations, while the error of measurement on the binary scale (alive or dead) analyzed with the animal linear model depends on the incidence. Estimates of the fixed effects and predictions of the random effects were obtained using the ASReml software (Gilmour et al., 2009). Pedigree used for analysis traced back four generations.

\subsubsection{Univariate linear animal model (Model 1)}

Model 1 (Univariate linear animal model): $\mathbf{y}=\mathbf{X b}+\mathbf{Z a}+\mathbf{W c}+\mathbf{e}$; where $\mathbf{y}$ is the vector of the phenotypic observations for survival; $\mathbf{X}$ is a design matrix for the fixed effects that links individual observations to the fixed effect class; $\mathbf{b}$ is the vector with the estimates of the fixed effects (sex, production week to account for age differences and weight of fish $12 \mathrm{~d}$ before challenge as a covariate); $\mathbf{Z}$ is an incidence matrix that links the observations to the animal

additive genetic effects; $\mathbf{a} \sim N\left(\mathbf{0}, \mathbf{A} \sigma_{a}^{2}\right)$ is a vector of random animal additive genetic effect; $\mathbf{W}$ is an incidence matrix that links the observations to the random environmental effects common 
to full-sibs; $\mathbf{c} \sim N\left(\mathbf{0}, \mathbf{I} \sigma_{c}^{2}\right)$ is a vector of random environmental effects common to full-sibs other than additive genetics; and $\mathbf{e}$ is a vector of random residuals. Finally, $\mathbf{A}$ is the numerator relationship matrix that describes the additive genetic relationship among all individuals included in $\mathbf{a}$, and $\mathbf{I}$ is an identity matrix of appropriate size.

For Model 1 the significance of the additive genetic effect and the effect common to fullsibs was assessed with the likelihood-ratio test (Lynch and Walsh, 1998): $L R=-2\left(\log L_{R}-\log L_{F}\right)$, where $\log L_{\mathrm{R}}$ is the $\log$ of the restricted likelihood of the reduced model (excluding each random effect at a time) and $\log \mathrm{L}_{\mathrm{F}}$ is the $\log$ of the restricted likelihood of the full model. The significance of LR was tested with a $\chi^{2}$ test with $\mathrm{df}=1$ (number of omitted variance components) and $\alpha=0.05$ for both random effects. For model 1, estimated heritability for survival was calculated as $h^{2}=\frac{\hat{\sigma}_{a}^{2}}{\hat{\sigma}_{a}^{2}+\hat{\sigma}_{c}^{2}+\hat{\sigma}_{e}^{2}}$, and the relative proportion of the environmental effects common to full-sibs as $c^{2}=\frac{\hat{\sigma}_{c}^{2}}{\hat{\sigma}_{a}^{2}+\hat{\sigma}_{c}^{2}+\hat{\sigma}_{e}^{2}}$.

\subsubsection{Univariate threshold sire-dam model (Model 2)}

Model 2 (Univariate threshold sire-dam model): $\lambda=\mathbf{X b}+\mathbf{Z}_{\mathbf{s}} \mathbf{a}_{\mathbf{s}}+\mathbf{Z}_{\mathbf{d}} \mathbf{a}_{\mathbf{d}}+\mathbf{W c}+\mathbf{e}$; where $\lambda \mathbf{i}$ is the underlying liability which is assumed to be associated to the binary observation of animal $\mathrm{i}(\mathrm{yi})$ such that $\lambda \mathrm{i} \leq 0$ gives yi $=0$, and $\lambda \mathrm{i}>0$ gives $\mathrm{yi}=1$. The vector $\lambda$ is a vector of all underlying liabilities. The variance components were estimated on the underlying scale, where residual variance $\left(\sigma_{E}^{2}\right.$ ) was restricted to 1.0 , implying that $\mathbf{e} \sim N(\mathbf{0}, \mathbf{I}) ; \mathbf{X}, \mathbf{b}, \mathbf{W}, \mathbf{c}$ and $\mathbf{I}$ are the same as described in Model $1 ; \mathbf{Z}_{\mathbf{S}}$ and $\mathbf{Z}_{\mathbf{D}}$ are incidence matrices linking observations to the sire and dam additive genetic effects; $\mathbf{a}_{\mathbf{S}} \sim N\left(\mathbf{0}, \mathbf{A} \sigma_{S}^{2}\right)$ and $\mathbf{a}_{\mathbf{D}} \sim N\left(\mathbf{0}, \mathbf{A} \sigma_{D}^{2}\right)$ are vectors of the random 
sire and dam additive genetic effects, respectively; sire and dam variance are assumed to be equal $\sigma_{S}^{2}=\sigma_{D}^{2}=\sigma_{u}^{2}$. The effect common to full-sibs (c) included the environmental tank/hapa effects caused by separate rearing of the families until tagging and potential dominance and maternal effects. For the individual family, these effects are confounded, and thus fitted as a single random effect. Log-likelihood ratio tests cannot be used to test significance of random effects in the threshold models. For Group1 a Probit link function was used to relate the linear predictor scale to the observation scale. Due to problems with convergence, a Logit link function was used for Group 2.

For model 2, estimated heritability for survival was calculated as $h^{2}=\frac{4 \hat{\sigma}_{u}^{2}}{2 \hat{\sigma}_{u}^{2}+\hat{\sigma}_{c}^{2}+\hat{\sigma}_{e}^{2}}$, and the relative proportion of the environmental effects common to full-sibs as $c^{2}=\frac{\hat{\sigma}_{c}^{2}}{2 \hat{\sigma}_{u}^{2}+\hat{\sigma}_{c}^{2}+\hat{\sigma}_{e}^{2}}$.

\section{Results}

\subsection{Mortality during acclimation}

During the $12 \mathrm{~d}$ acclimation period at the AAHRU, a total of 189 fish died, which accounted for approximately $11 \%$ of the fish in Group 1 and $4 \%$ in Group 2. Most acclimation mortalities occurred during the first three days after transportation (Fig. 1). No mortalities were recorded in the large tank (Group 2) after $6 \mathrm{~d}$ post transportation, indicating fish were fully acclimated.

\subsection{Streptococcus iniae challenge}

Accumulated mortality at the end of the challenge experiment was $60 \%$ for the fish in 
Group 1 that were challenged by injection, and $6.4 \%$ for fish in Group 2 challenged by cohabitation (Fig. 2). Most of the fish from Group 1 died on the $1^{\text {st }}$ and $2^{\text {nd }}$ day after injection, and in the remaining $37 \mathrm{~d}$ mortalities were low. Brain tissue was cultured from approximately $20 \%$ of the fish that died during the trial and $98 \%(180 / 183)$ of these yielded pure cultures of $S$. iniae. Most of the fish challenged by cohabitation survived, consistent with a pilot study where 0 $-12 \%$ mortality was observed in the cohabitants regardless of the bacterial dose and the number of fish injected to serve as shedder animals (data not shown). The water quality parameters during the course of the challenge were determined as follows (mean \pm standard error): $\mathrm{DO}=$ $3.58 \pm 0.09 \mathrm{mg} \mathrm{L}^{-1} ;$ temperature $=26.2 \pm 0.07^{\circ} \mathrm{C} ;$ nitrate $=1.43 \pm \mathrm{mg} \mathrm{L}^{-1} ;$ ammonia $=2.89 \pm 0.4$ $\mathrm{mg} \mathrm{L}^{-1}$; hardness $=140 \pm 3.4 \mathrm{mg} \mathrm{L}^{-1}$; and alkalinity $=135 \pm 5 \mathrm{mg} \mathrm{L}^{-1}$.

The results revealed high variation between the mean family survival of the fish injected with S. iniae (range from 0\% to 100\%, CV 69\%; Fig. 3A). On stark contrast, there was low variation between the mean family survival of the fish challenged by cohabitation (range 60\%100\%, CV 10\%; Fig. 3B). The Pearson correlation between the mean family survival of Group 1 and Group 2 was positive but of low magnitude $(r=0.31)$.

At the termination of the challenge, all survivors were examined for clinical signs of disease. Overall, eight percent of the surviving fish showed pustules, but a higher percentage of pustules were observed on fish challenged by injection (16.6\% for Group 1) when compared with fish that were challenged through cohabitation (4.8\% for Group 2). Approximately seventy percent of the surviving fish exhibited cloudy eyes (73.3\% for Group 1, 65.7\% for Group 2). Distribution of clinical signs of disease observed in survivors from Group 1 and Group 2 are shown in Fig. 4. 


\subsection{Fixed effects}

None of the fixed effects fitted in the models (sex, production week, weight at transport) were found to be statistically significant $(\mathrm{P}>0.05$, Wald $\mathrm{F}$ statistics).

\subsection{Additive genetic effects}

The results showed a strong additive genetic component for survival in fish injected with S. iniae (Group 1; Table 1). The heritability estimate on the observed scale was $0.42 \pm 0.07$ (Model 1), and higher on the underlying scale $(0.58 \pm 0.09$; Model 2$)$, as expected. The additive genetic effect was significantly different from zero $(P<0.05$; Log-likelihood ratio test Model 1$)$.

In contrast, a very low additive genetic component was found for survival in fish challenged by cohabitation (Group 2; Table 1). On the observed scale (Model 1), the heritability estimate was $0.06 \pm 0.07$ and additive genetic effect for survival was not significantly different from zero $(P>0.05$; Log-likelihood ratio test Model 1). Heritability for the threshold model (Model 2) was higher $(0.54 \pm 0.59)$, however with a standard error of the same magnitude suggesting that the heritability is not statistically different from zero. Results suggest no statistically significant additive genetic variation for survival in fish challenged with $S$. iniae by cohabitation.

\subsection{Environmental effects common to full-sibs}

The environmental effect common to full-sibs was not significant for either fish challenged with $S$. iniae by injection (Group 1) or for the fish challenged by cohabitation (Group 2) $(P>0.05 ;$ Log-likelihood ratio test Model 1). The environmental effect common to full-sibs as a proportion of phenotypic variance $\left(\mathrm{c}^{2}\right)$ was zero for Group 1 (in both models) and low and 
not significant for Group 2 (Table 1).

\section{Discussion}

Although tilapia production is increasing worldwide, mortality resulting from climatic events and diseases have limited production and the growth of the industry (Thodesen et al., 2013). Streptococcosis caused by Streptococcus iniae (Shoemaker et al., 2001) is one important bacterial pathogen impacting tilapia production through disease losses. Resistance against bacterial, viral and parasitic pathogens in fish can be heritable (Ødegård et al., 2011); thus, selective breeding for resistance to $S$. iniae provides a complementary approach for limiting the impact of streptococcosis if there is an additive genetic component to variation in resistance.

Immersion and/or cohabitation disease models are generally preferred for fish disease research, including phenotyping families for resistance to disease. In preliminary studies, attempts were made to develop a cohabitation disease model to mimic natural exposure (data not shown). In these studies, different doses of $S$. iniae were tested in conjunction with different percentages of injected fish (shedders) and naïve fish (cohabitants) in the same tank. Regardless of the number of shedder fish (20 or $50 \%$ of the population) and bacterial doses used, limited transmission of disease to cohabitants was noted. For example, ip injection of shedder fish with 1 $\times 10^{8} \mathrm{CFU} /$ fish resulted in $60-70 \%$ mortality in the shedder fish, whereas only $8-10 \%$ mortality occurred in the naïve fish housed in the same tank. Although these preliminary studies suggested limited mortality in cohabitant fish, a similar experimental design was used for the phenotyping of all families in anticipation of greater disease transmission with larger numbers of fish and higher densities in the 5,500 L tank. The challenge was designed with the intent to utilize fish injected with S. iniae (Group 1) as shedder fish to transfer the bacterium to 
cohabitated fish (Group 2) and determine the mortality of each family by both injection and cohabitation. However, results of the large tank study largely confirmed the preliminary study results in that $60 \%$ mortality was recorded in the fish injected i.p. (Group 1) and only 6.4\% mortality observed in the naïve cohabitated fish (Group 2).

Analysis of the mortality data from fish that were ip injected with S. iniae (Group 1), demonstrated substantial additive genetic variation in survival of Nile tilapia to $S$. iniae challenge. The estimated heritability of post-challenge survival in Group 1 was $0.42 \pm 0.07$ on the observed binary scale (Model 1) and $0.58 \pm 0.09$ on the underlying liability scale (Model 2), derived from fitting a linear animal model and a sire-dam threshold model, respectively. Ødegård et al. (2011) suggested the simplest statistical model for analysis of challenge data are the crosssectional models if certain assumptions are met (i.e, alive/dead and 50\% end point mortality). In this study, the cumulative end of test mortality was $60 \%$ for Group 1; therefore, a simple cross sectional model such as Model 1 is considered appropriate since phenotypic variance of a binary trait is maximized at $50 \%$ of cumulative mortality and thus difference between results in the observed and underlying scale are consistent. In contrast, analysis of the mortality data obtained from the cohabitant fish (Group 2) suggested no additive genetic variation in survival to $S$. iniae challenge. On the observed binary scale (Model 1), the heritability estimate was $0.06 \pm 0.07$ and for the threshold model (Model 2) and $0.54 \pm 0.59$ on the underlying liability scale (Model 2). This finding was not surprising, given the low mortalities in Group 2 and lack of variation between Group 2 mean family survivals. For both groups, the environmental effect common to full-sibs was not significant, indicating that initial separate rearing of individual families prior to tagging, maternal or epistatic effects do not affect the survival to $S$. iniae, as was done in this experiment. 
These results suggest good prospect for genetic improvement based on $S$. iniae challenge testing using ip injection. Although injection disease models bypass the natural immune barriers, this route of challenge has demonstrated additive genetic variation for bacterial cold water disease caused by Flavobacterium psychrophilum in rainbow trout (Oncorhynchus mykiss) (Silverstein et al., 2009; Leeds et al., 2010), cold water vibriosis (Vibrio salmonicida) and bacterial kidney disease (Renibacterium salmoninarum) in Atlantic salmon Salmo salar (Gjedrem and Gjøen, 1995), Vibrio anguillarum in Atlantic salmon (Gjøen et al., 1997), Piscirickettsia salmonis in Atlantic salmon (Yañez et al., 2013), and infectious salmon anemia virus in Atlantic salmon (Ødegård et al., 2007). Importantly, use of the injection challenge model in rainbow trout for the $F$. psychrophilum selective breeding program has translated to enhanced performance of selectively bred fish in field trials, where the fish were exposed to the pathogen under natural conditions (Wiens et al., 2013). It will be important to validate the relationship between the survival of tilapia in the challenge test for $S$. iniae (via ip injection) and survival to S. iniae outbreaks under field conditions. To validate this, fish from the same families (or experimental groups) must be challenged simultaneously under controlled conditions and under commercial field conditions. However, in a practical sense this can be difficult to achieve because you cannot always ensure outbreak of disease in the field.

The results demonstrated substantial additive genetic variation in resistance to $S$. iniae when fish are challenged by injection, and suggest promise in genetic improvement of tilapia for resistance to $S$. iniae by use of selective breeding. The estimates were based on a relatively small family size (on average nine fish per family). Future testing of tilapia from the same population will include a higher number of fish per family to allow for higher accuracy of estimated family breeding values. Additionally, tilapia will be challenged with S. agalactiae, another important 
tilapia pathogen (Delannoy et al., 2014), to determine if there is a genetic correlation between the resistance of tilapia to both Streptococcus species. Evenhuis et al. (2015) recently demonstrated that selection of rainbow trout for resistance to $F$. psychrophilum resulted in a favorable genetic correlation in resistance to another bacterium in the same genus, $F$. columnare. Research is also in progress to determine the potential for marker assisted selection in which selection is based on marker genotypes alone or in combination with phenotypic information on the tilapia families. Such an approach may further improve the accuracy and response to selection for resistance to $S$. iniae. The long term goal of this research is to provide farmers with a more resistant stock of tilapia as an additional management tool for reducing production losses due to Streptococcus spp. 


\section{Acknowledgments}

The authors acknowledge Spring Genetics for providing the Nile tilapia under a Material Transfer Agreement for this research and for the technical assistance of José Ospina and Hideyoshi Segovia Uno. The authors also acknowledge the technical support of Paige Mumma, Curtis Day, Ning Qin, and Jana Mladek of USDA-ARS during the challenge test trials conducted in Auburn. This research was supported by USDA-ARS CRIS Project No. 6010-32000-026-00D, Pathogen characterization, host immune response and development of strategies to reduce losses to disease in aquaculture. Mention of trade names or commercial products in this publication is solely for the purpose of providing specific information and does not imply recommendation or endorsement by the United States Department of Agriculture. 


\section{References}

Agnew, W., Barnes, A.C., 2007. Streptococcus iniae: An aquatic pathogen of global veterinary significance and a challenging candidate for reliable vaccination. Vet. Microbiol. 122, 115.

Bentsen, H.B., Eknath, A.E., Palada-de Vera, M.S., Danting, J.C., Bolivar, H.L., Reyes, R.A., Dionisio, E.E., Longalong, F.M., Circa, A.V., Tayamen, M.M., Gjerde, B., 1998. Genetic improvement of farmed tilapias: growth performance in a complete diallel experiment with eight strains of Oreochromis niloticus. Aquaculture 160, 145-173.

Bentsen, H.B., Gjerde, B., Nguyen, N.H., Rye, M., Ponzoni, R.W., Palada-de Vera M.S., Bolivar, H.L., Velasco, R., Danting, J.C., Dionisio, E.E., Longalong, F.M., Reyes, R.A., Abella, T.A., Tayamen, M.M., Eknath, A.E., 2012. Genetic improvement of farmed tilapias: Genetic parameters for body weight at harvest in Nile tilapia (Oreochromis niloticus) during five generations of testing in multiple environments. Aquaculture 338$341,56-65$.

Delannoy, C., Crumlish, M., Fontaine, M.C., Pollock, J., Foster, G., Dagleish, M.P., Turnbull, J.F., Zadoks, R.N. 2013. Human Streptococcus agalactiae strains in aquatic mammals and fish. BMC Microbiol., 13:41.

Eldar, A., Horovitcz, A., Bercovier, H. 1997. Development and efficacy of a vaccine against Streptococcus iniae infection in farmed rainbow trout. Vet. Immunol. Immunopathol. 56, $175-183$.

Evenhuis, J.P., Leeds, T.D., Marancik, D.P., LaPatra, S.E., Wiens, G.D. 2015. Rainbow trout (Oncorhynchus mykiss) resistance to columnaris disease is heritable and favorably correlated with bacterial cold water disease resistance. J. Anim. Sci. 93, 1546-1554. 
FAO, 2014. Fishery and Aquaculture Statistics 2012, Rome, Italy, pp. 105.

Fitzsimmons, K., Martinez-Garcia, R., Gonzales-Alanis, P. 2011. Why tilapia is becoming the most important food fish on the planet? In: Liu, L.P., Fitzsimmons, K. (Eds.), Proceedings of the 9th International Symposium on Tilapia in Aquaculture. Shanghai, , pp. 1-8 (409 pp.)..

Gaunt, P.S., Endris, R., McGinnis, A., Baumgartner, W., Camus, A., Steadman, J., Sweeney, D., Sun, F. 2010. Determination of florfenicol dose rate in feed for control of mortality in Nile tilapia infected with Streptococcus iniae. J. Aquat. Anim. Health 22, 158-166.

Gjedrem, T., Gjøen, H.M. 1995. Genetic variation in susceptibility of Atlantic salmon, Salmo salar L., to furunculosis, BKD and cold water vibriosis. Aquaculture Res. 26, 129-134.

Gjerde, B., Mengistu, S.B, Ødegård, J., Johansen, H., Soza, D. 2012. Quantitative genetics of body weight, fillet weight and fillet yield in Nile tilapia (Oreochromis niloticus). Aquaculture 342-343, 117-124.

Gjøen, H.M., Refstie, T., Ulla, O., Gjerde, B. 1997. Genetic correlations between survival of Atlantic salmon in challenge and fieled tests. Aquaculture 158, 277-288.

Gilmour, A.R., Gogel, B.J., Cullis, B.R., Welham, S.J., Thompson, R., 2009. ASReml User Guide, Release 3.0. VSN, Hemel Hempstead, UK.

LaFrentz, B.R., Shoemaker, C.A., Klesius, P.H. 2011. Immunoproteomic analysis of the antibody response obtained in Nile tilapia following vaccination with a Streptococcus iniae vaccine. Vet. Microbiol. 152, 346-352.

Leeds, T.D., Silverstein, J.T., Weber, G.M., Vallejo, R.L., Palti, Y., Rexroad III, C.E., Evenhuis, J., Hadidi, S., Welch, T.J., Wiens, G.D. 2010. Response to selection for bacterial cold water disease resistance in rainbow trout. J. Anim. Sci. 88, 1936-1946. 
Locke, J.B., Vicknair, M.R., Ostland, V.E., Nizet, V., Buchanan, J.T.. 2010. Evaluation of Streptococcus iniae killed bacterin and live attenuated vaccines in hybrid striped bass through injection and bath immersion. Dis. Aquat. Org. 89, 117-123.

Lynch, M., Walsh, B., 1998. Genetics and Analysis of Quantitative Traits. Sinauer Associates, Inc., Massachusetts. USA, 980 pp.

Mata, A.I., Blanco, M.M., Domínguez, L., Fernández-Garayzábal, J.F., Gibello, A. 2004. Development of a PCR assay for Streptococcus iniae based on the lactate oxidase (lctO) gene with potential diagnostic value. Vet. Microbiol. 101, 109-116.

Ødegård, J., Olesen I., Gjerde B., Klemetsdal, G. 2007. Positive genetic correlation between resistance to bacterial (furunculosis) and viral (infectious salmon anemia) diseases in farmed Atlantic dalmon (Salmo salar). Aquaculture 271, 173-177.

Ødegård, J., Baranski, M., Gjerde, B., Gjedrem, T. 2011. Methodology for genetic evaluation of disease resistance in aquaculture species: Challenges and future prospects. Aquaculture Res. 42, 103-114.

Pier, G.B., Madin, S.H. 1976. Streptococcus iniae sp. nov., a beta hemolytic streptococcus isolated from an Amazon freshwater dolphin, Inia geoffrensis. Int. J. Syst. Bacteriol. 26, $545-553$.

Silverstein, J.T., Vallejo, R.L., Palti, Y., Leeds, T.D., Rexroad III, C.E., Welch, T.J., Wiens, G.D., Ducrocq, V. 2009. Rainbow trout resistance to bacterial cold-water disease is moderately heritable and is not adversely correlated with growth. J. Anim. Sci. 87, 860867. 
Shoemaker, C.A., Evans, J.J., Klesius, P.H. 2000. Density and dose: factors affecting mortality of Streptococcus iniae infected tilapia (Oreochromis niloticus). Aquaculture 188, 229235.

Shoemaker, C.A., Klesius, P.H., Evans, J.J. 2001. Prevalence of Streptococcus iniae in tilapia, hybrid striped bass, and channel catfish on commercial fish farms in the United States. Am. J. Vet. Res. 62, 174-177.

Shoemaker, C.A., Xu, D., Evans, J.J., Klesius, P.H. 2006. Parasites and diseases. In: Lim, C., Webster, C.D. (Eds.), Tilpaia Biology, Culture and Nutrition. Haworth Press, Inc., New York, pp. 561-582.

Shoemaker, C.A., LaFrentz, B.R., Klesius, P.H., Evans, J.J. 2010. Protection against heterologous Streptococcus iniae isolates using a modified bacterin vaccine in Nile tilapia, Oreochromis niloticus (L.). J. Fish Dis. 33, 537-544.

Thodesen, J., Rye, M., Wang, Y.-X., Li, S.-J., Bentsen, H.B., Gjedrem, T. 2013. Genetic improvement of tilapias in China: Genetic parameters and selection responses in growth, pond survival and cold-water tolerance of blue tilapia (Oreochromis aureus) after four generations of multi-trait selection. Aquaculture 396-399, 32-42.

Wiens, G.D., LaPatra, S.E., Welch, T.J., Evenhuis, J.P., Rexroad III, C.E., Leeds, T.D. 2013. Onfarm performance of rainbow trout (Oncorhynchus mykiss) selectively bred for resistance to bacterial cold water disease: Effect of rearing environment on survival phenotype. Aquaculture 388-391, 128-136.

Yáñez, J.M., Bangera, R., Lhorente, J.P., Oyarzún, M., Neira, R. 2013. Quantitative genetic variation of resistance against Piscirickettsia salmonis in Atlantic salmon (Salmo salar). Aquaculture 414-415, 155-159. 


\section{Table 1}

Survival variance components, estimated heritability $\left(\mathrm{h}^{2} \pm \mathrm{se}\right)$ and effect common to full-sibs $\left(\mathrm{c}^{2}\right.$ \pm se) for the univariate linear animal model (Model 1) and the univariate threshold sire-dam model (Model 2).

\begin{tabular}{|c|c|c|c|c|}
\hline \multirow[b]{2}{*}{ Parameter } & \multicolumn{2}{|c|}{ Model 1 (observed scale) } & \multicolumn{2}{|c|}{ Model 2 (underlying scale) } \\
\hline & G1 & G2 & $\mathrm{G}_{1}^{*}$ & $\mathrm{G}^{* *}$ \\
\hline$\sigma^{2}$ sire & - & - & 0.207 & 0.232 \\
\hline$\sigma^{2} \mathrm{dam}$ & - & - & 0.207 & 0.232 \\
\hline$\sigma^{2}$ additive & 0.100 & 0.004 & 0.826 & 0.927 \\
\hline$\sigma^{2}$ common env. & 0.000 & 0.002 & 0.000 & 0.257 \\
\hline$\sigma^{2} e$ & 0.139 & 0.055 & 1.000 & 1.000 \\
\hline$h^{2} \pm s e$ & $0.42 \pm 0.07$ & $0.06 \pm 0.07$ & $0.58 \pm 0.09$ & $0.54 \pm 0.59$ \\
\hline$c^{2} \pm s e$ & $0.00 \pm 0.00$ & $0.03 \pm 0.03$ & $0.00 \pm 0.00$ & $0.15 \pm 0.29$ \\
\hline
\end{tabular}




\section{Figure Legends}

Fig. 1. The number of dead Nile tilapia recorded from Group $1(\bullet)$ and Group $2(\mathbf{\square})$ following transportation. Fish were acclimated for $12 \mathrm{~d}$ prior to bacterial challenge.

Fig. 2. Cumulative percent mortality in Nile tilapia following challenge with Streptococcus iniae by intraperitoneal injection (•; Group 1) or by cohabitation ( $\bullet$; Group 2).

Fig. 3. The number of fish per family (line; right y-axis) and mean survival (bars; left y-axis) of 143 Nile tilapia families following challenge with Streptococcus iniae by intraperitoneal injection (A; Group 1) or cohabitation (B; Group 2). Families with less than five fish at the onset of the bacterial challenge were excluded from the data analysis (see section 2.5. Data Analysis).

Fig. 4. Percentage of clinical signs of streptococcosis observed in Nile tilapia surviving challenge with Streptococcus iniae by intraperitoneal injection (A; Group 1) or cohabitation (B; Group 2). 


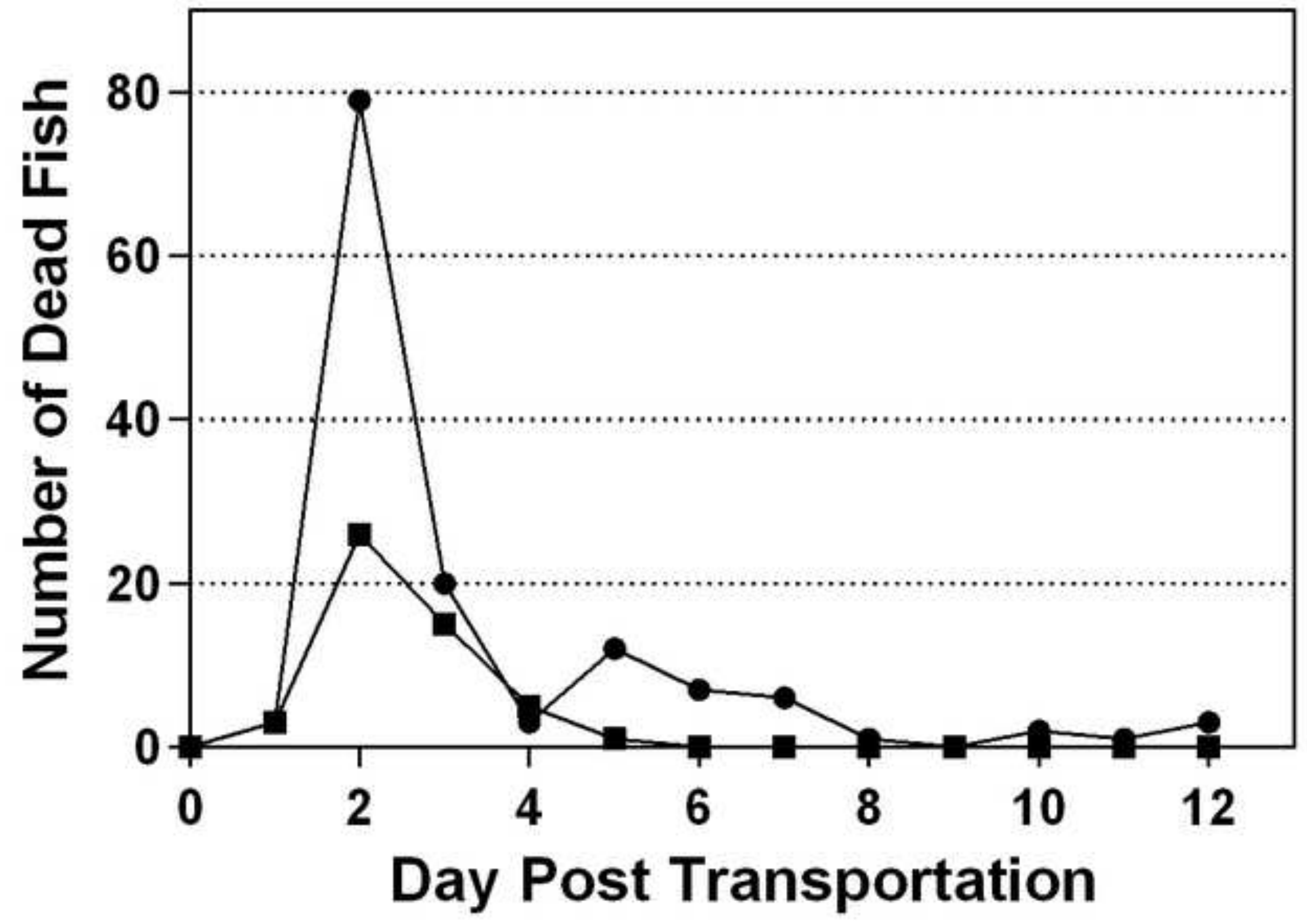




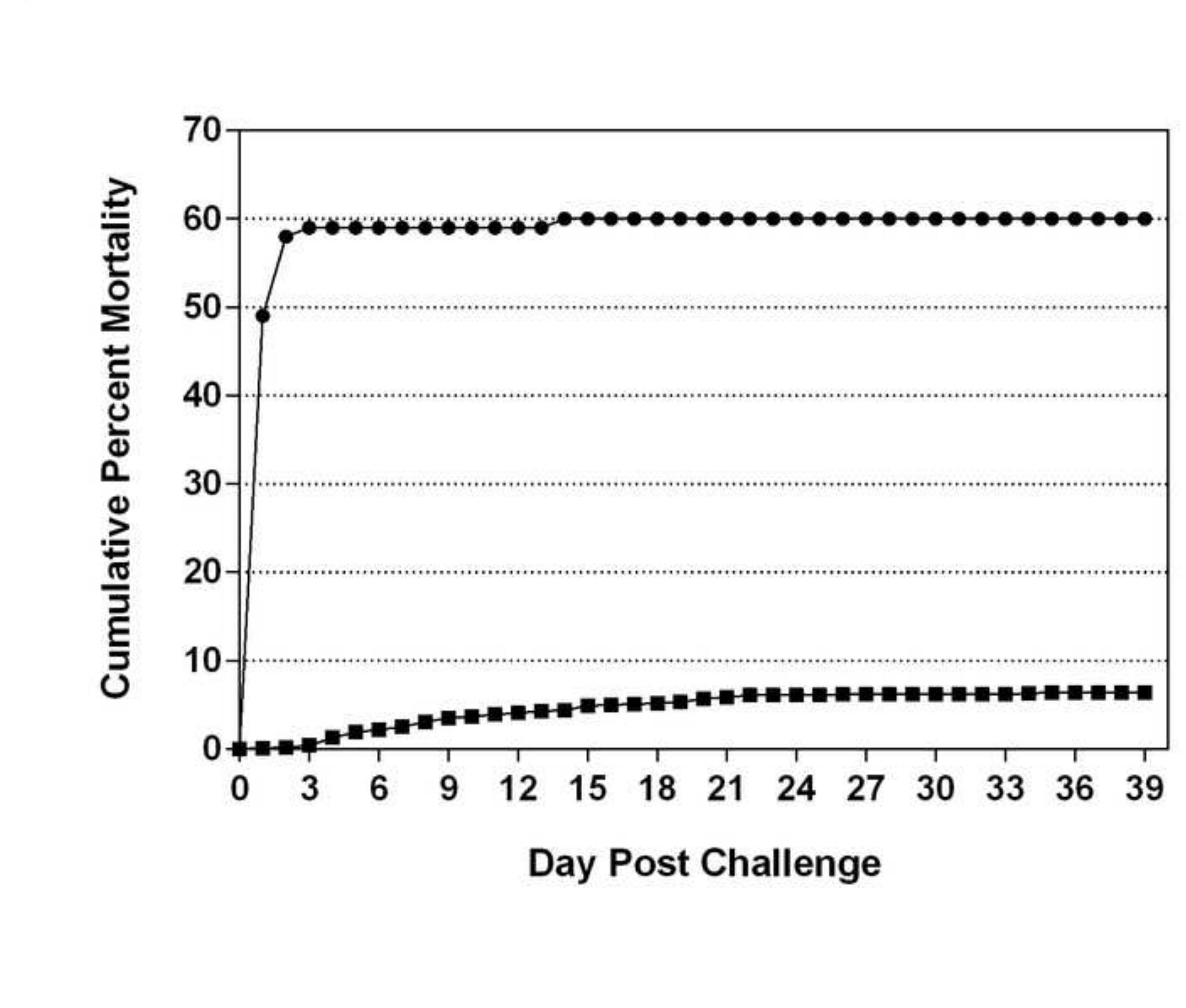

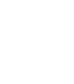

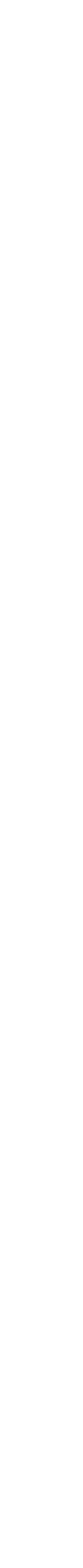

C

.

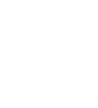

.
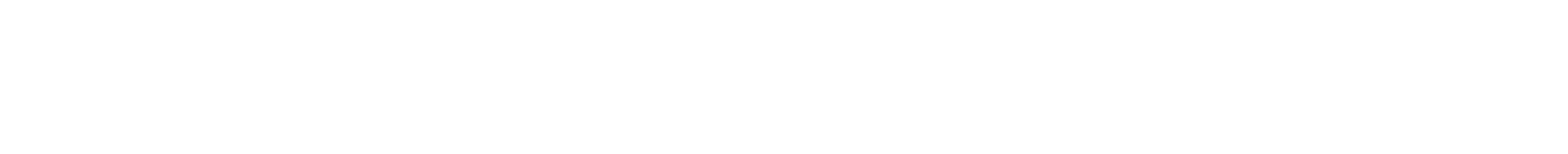


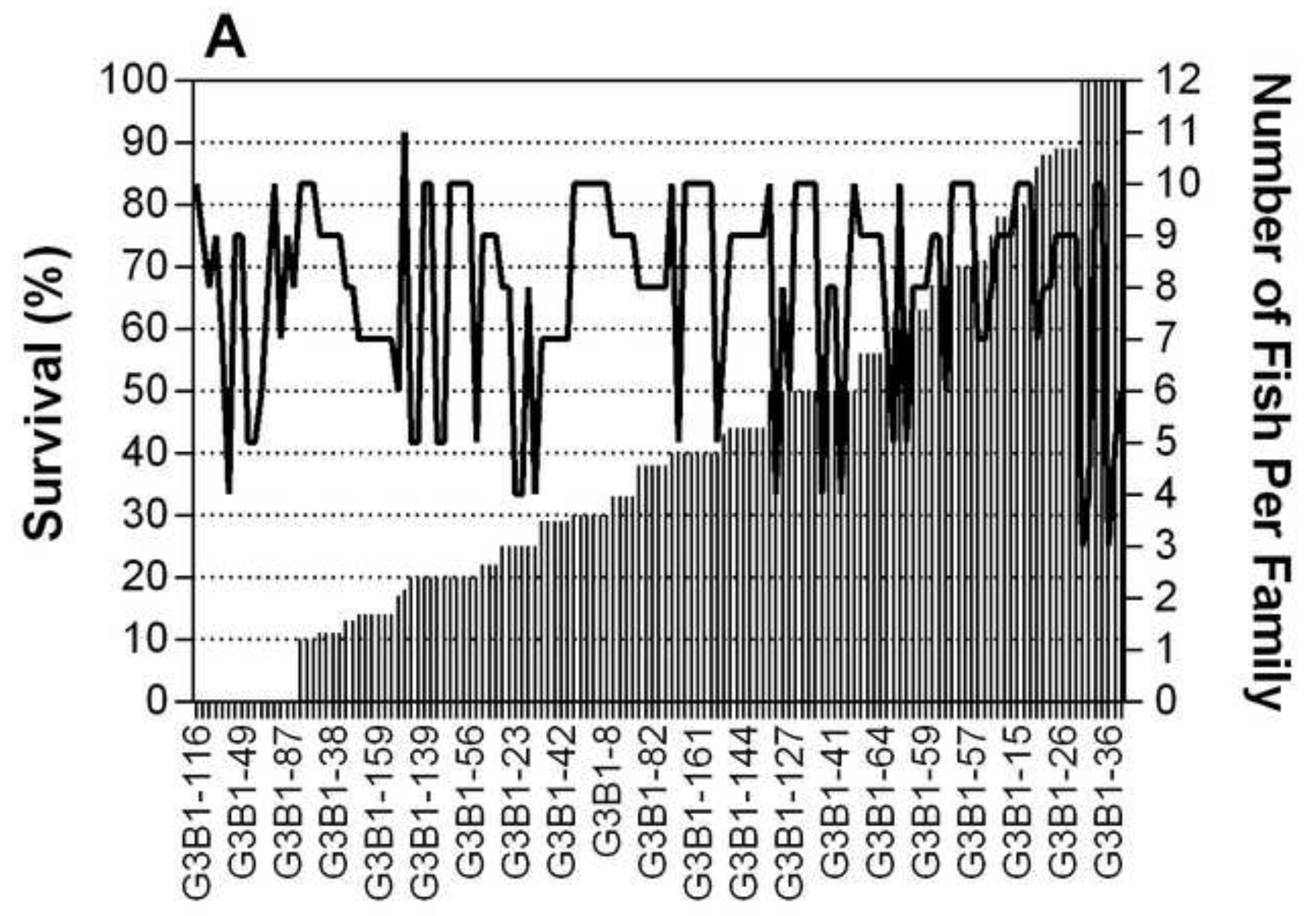

Families 


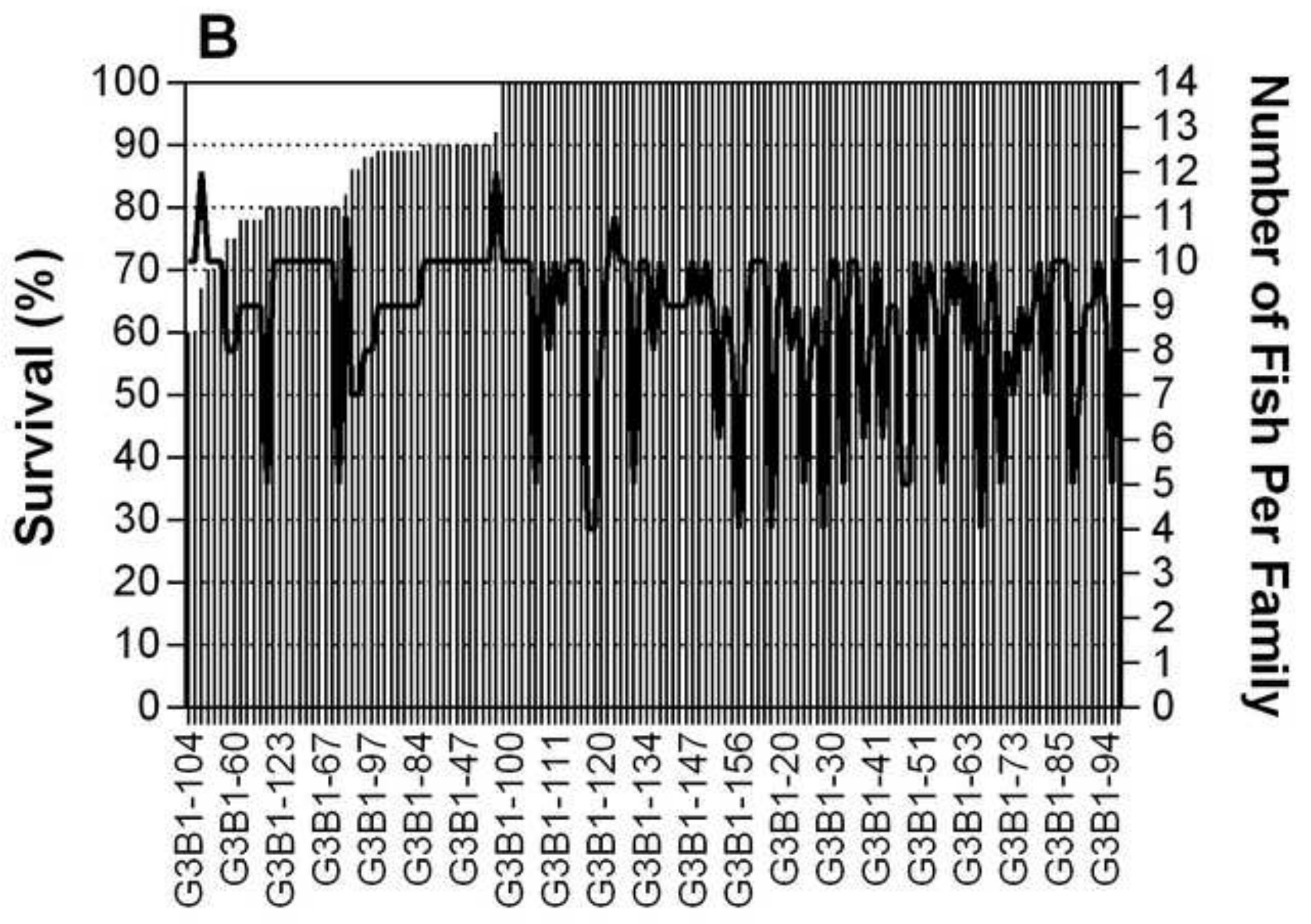

Families 


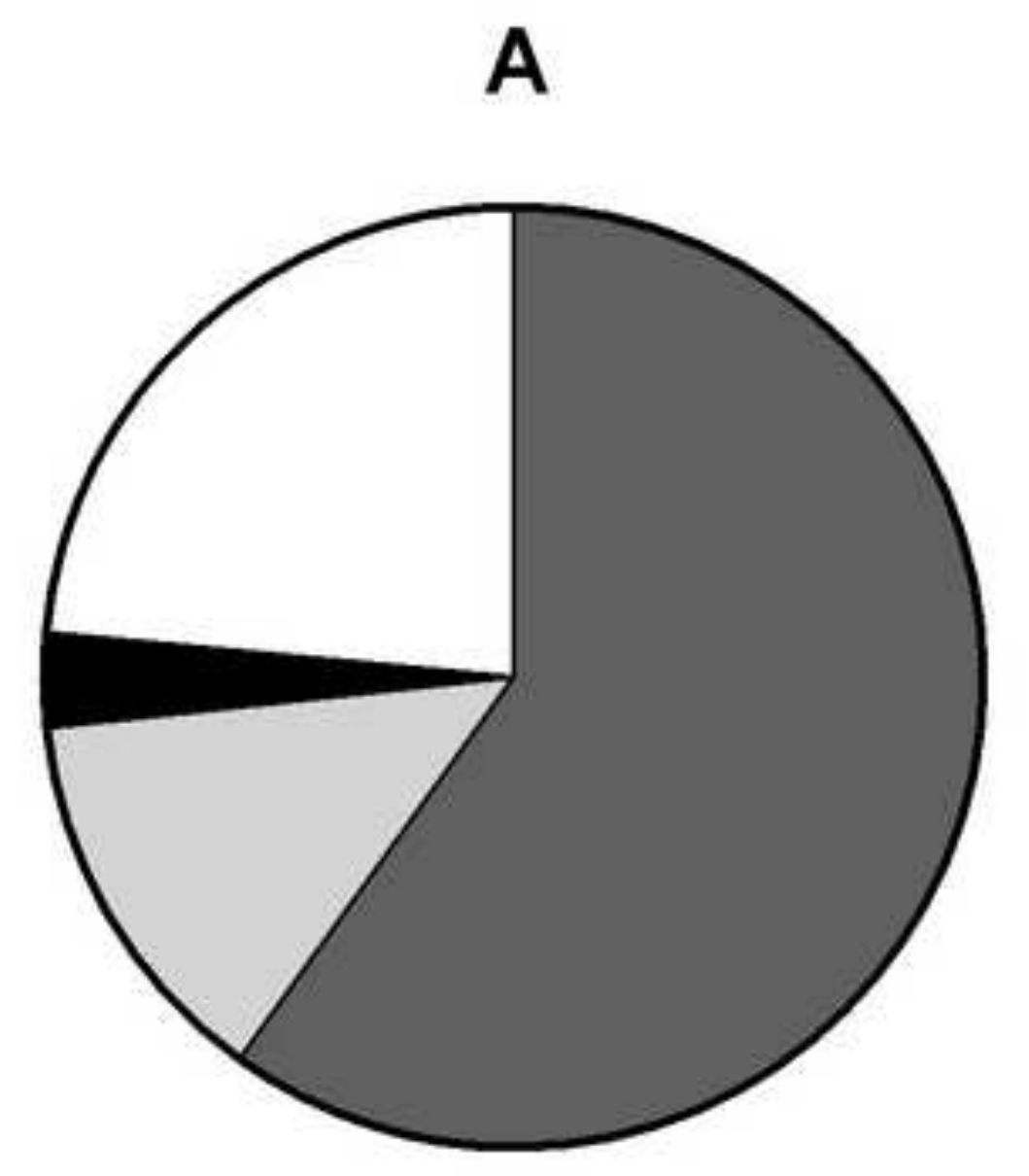

$\square$ Cloudy Eye (59.9\%)

$\square$ Cloudy Eye and Pustules (13.4\%)

- Pustules (3.2\%)

$\square$ No Clinical Signs (23.5\%)

\section{$N=464$ Survivors}


B

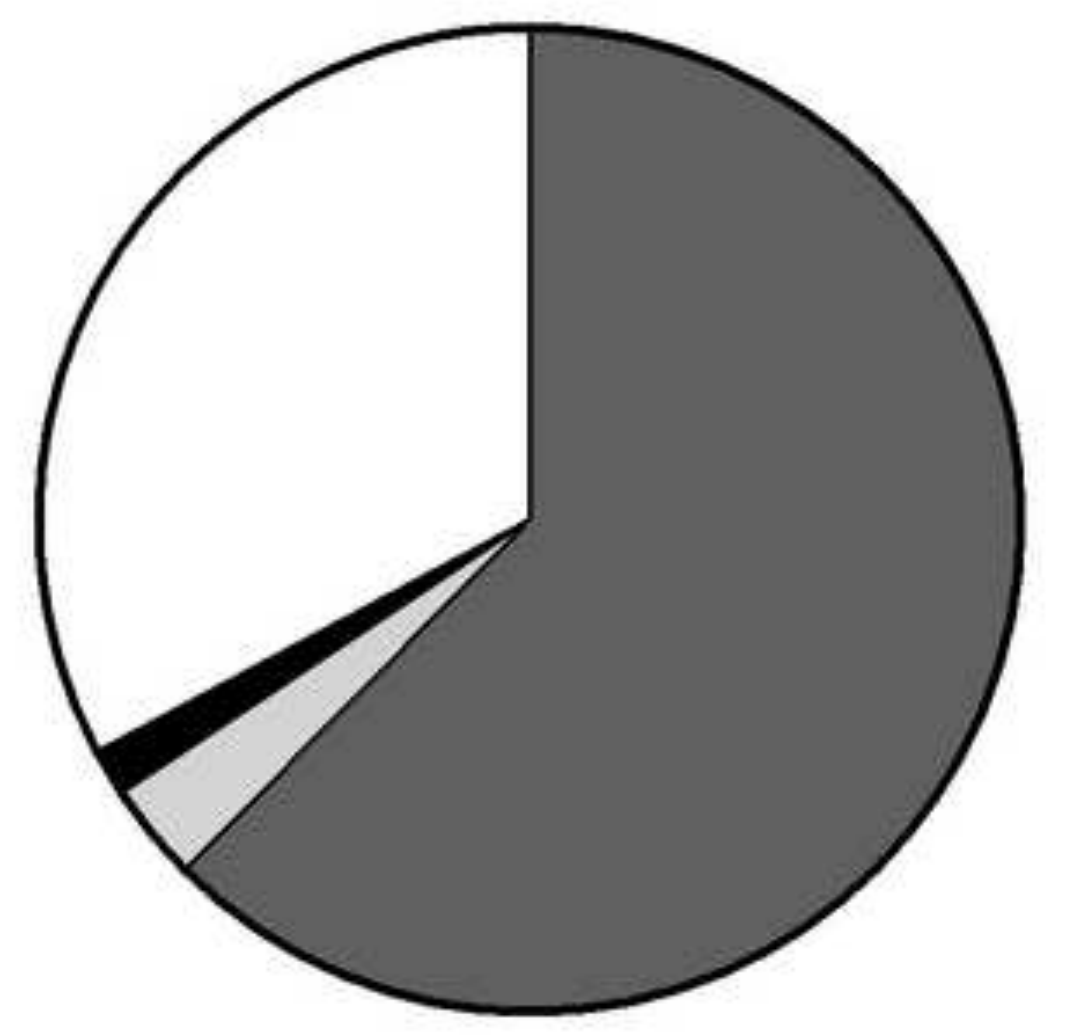

$\square$ Cloudy Eye (62.4\%)

$\square$ Cloudy Eye and Pustules (3.3\%)

- Pustules (1.5\%)

$\square$ No Clinical Signs (32.8\%)

$N=1170$ Survivors 